\title{
Parkinson's Disease Patients with Dopamine Dysregulation Syndrome-Editorial Review
}

\section{Hristina Popovska* and Igor Petrov}

Department of Neurology, University SS. Cyril and Methodius, Macedonia

*Corresponding Author: Hristina Popovska, Department of Neurology, University SS. Cyril and Methodius, Macedonia.
Received: July 29, 2020

Published: August 26, 2020

(C) All rights are reserved by Hristina

Popovska and Igor Petrov.

\begin{abstract}
Over the past decade, it has been identified a novel disease entity in patients with Parkinson's disease, impulse-compulsive spectrum disorder. Dopamine dysregulation syndrome (DDS) included in this group, is a syndrome characterized for a patients treated with dopaminergic therapy, especially Levodopa. The phenomenology of this syndrome is in a close relation with the current understanding of the basal ganglia function and its impact on a long-term management in patients with Parkinson's disease.

Patients treated with dopaminergic therapy, develop cognitive and behavioral disturbances besides motor symptoms. The PD therapy which basically is dopamine replacement therapy (DRT) can relieve the motor symptoms. On the other hand, recent studies show that the same therapy can be a cause of behavioral alterations, especially when it is abused by the patients. It is very important to differentiate the psychological symptoms whether they are related to the disease itself or are a side effect from the therapy.
\end{abstract}

Keywords: Dopamine Dysregulation Syndrome; Parkinson's Disease; Psychiatric Symptoms

\section{Introduction}

Parkinson's disease (PD) is considered an expression of a diffuse degeneration, affecting the peripheral and central nervous system. PD is regarded a progressive alfa-synucleinopathic neurodegenerative disease, manifesting characteristically with both appendicular (hypokinesia, bradykinesia, rigidity and tremor) and/ or axial (gait impairment, postural changes and postural instability) motor symptoms, though accompanied and in some cases even preceded by a multitude of non-motor signs and symptoms. Non-motor symptoms comprise autonomic dysfunctions such as cardiovascular, urogenital and gastro-intestinal manifestations, sleep-wake disorders (sleep fragmentation, insomnia, excessive daytime somnolence, sleep attacks, REM sleep behavior disorder), sensory disorders (hyposmia or pain, impaired color vision, vestibular deficits), and neuropsychiatric disorders including mood disturbances, anxiety, depression, cognitive deficits, dementia and psychosis, impulsive-compulsive spectrum disorders.
In PD, there is increasing evidence for disorders in the impulsive-compulsive spectrum, related to the disease itself, to the pharmacological management of the disease, or to both. These disorders comprise dopamine deficiency syndrome (with immediate reward seeking behavior), dopamine dependency syndrome (with addictive behavior), dopamine dysregulation syndrome (with both addictive behavior and stereotyped behavior) and impulse control disorders (pathological gambling, compulsive shopping, binge eating and hypersexuality) $[3,4]$.

\section{Dopamine, dopaminergic pathways}

Dopamine (DA) is a diverse and important neurotransmitter in the human body. It is a catecholamine neurotransmitter that is a precursor to the synthesis of the neurotransmitter norepinephrine. DA is synthesized from tyrosine by a two step process, where tyrosine hydroxylase is the rate-limiting enzyme in the reaction. 3,4-dihydroxyphenylalanine (DOPA) is the precursor to DA. Whether or 
not dopamine acts as an excitatory or inhibitory neurotransmitter, depends on the receptor it is acting at (Figure 1).

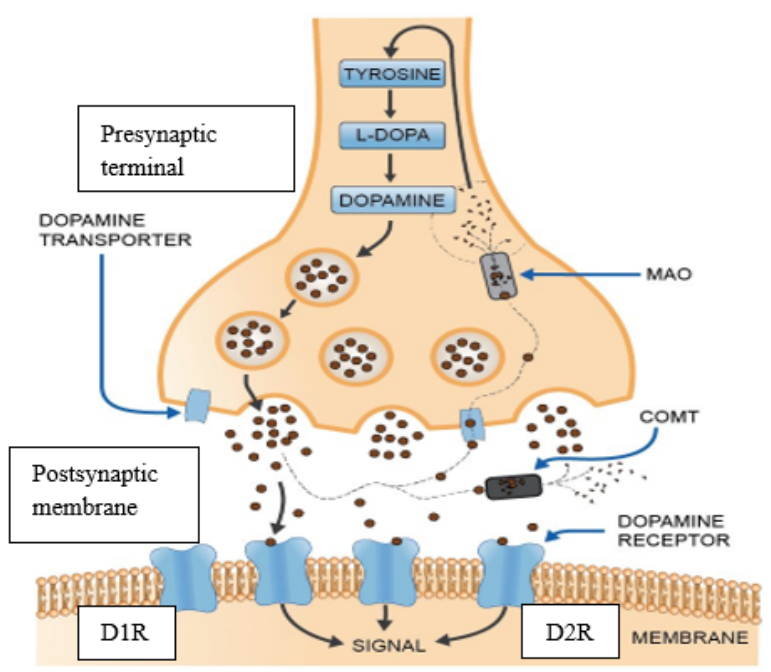

Figure 1: Diagram of a dopaminergic synapse. Enlarged view of a typical dopaminergic synapse. The presynaptic terminal and the postsynaptic membrane/neuron. DOPA: 3,4 -dihydroxyphenylalanine; MAO: Monoamine Oxidase; COMT:

Catechol-0-Methyl Transferase; D1R: Type 1 Dopamine

Receptor; D2R: Type 2 Dopamine Receptor.

There are two families of dopamine receptors: D1 and D2. The D1 to D5 subtypes of the D1 family of dopamine receptors are metabotropic receptors coupled to $G$ proteins which stimulate adenylate cyclase. D2 family of dopamine receptors makes inhibition of adenylate cyclase and suppression of cAMP production. D1 receptors are primarily found on neurons involved in the direct pathway and D2 receptors on neurons involved in the indirect pathway. Both, D1 and D2 receptors are found in neurons in the striatum (basal ganglia), as well as in the neurons of the cortex and the limbic system. D2 receptors are found in the pituitary gland.

Dopamine pathways (Figure 2) are neuronal connections in which dopamine travels to areas of the brain and body to convey important information. There are four major DA projections in the central nervous system. The mesolimbic and mesocortical projections originate in the ventral tegmental area (VTA) (VTA is a dopamine-rich nucleus that covers part of the midbrain) and project both to the ventral striatum and areas in the prefrontal cortex, respectively. The nigrostriatal pathway originates in the substantia nigra and projects to the dorsal striatum. The final system is the tuberoinfundibular system which projects from the hypothalamus to the pituitary gland.

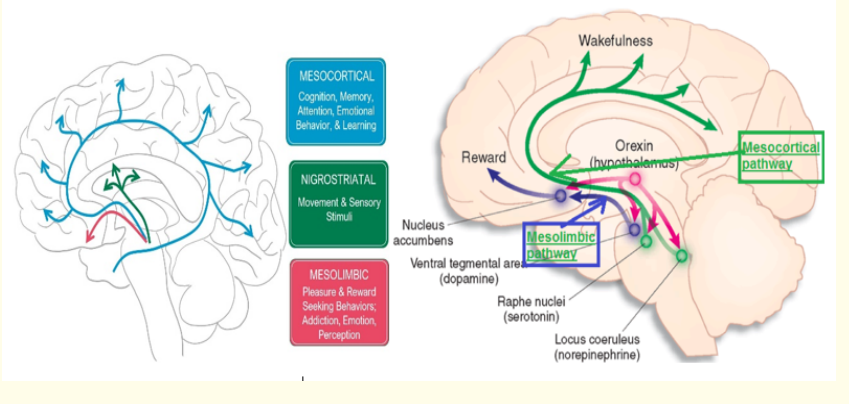

Figure 2: Dopaminergic pathways.

The reward system, physiology and pathophysiology

The mesolimbic pathway is well known as a reward circuitry, regulated by neurotransmitter interactions, and net release of the substance dopamine in the nucleus accumbens. This brain reward cascade, leading to reward, involves the inter-relationship of at least four important neurochemical pathways: serotonergic (5-HT), enkephalinergic (Enk), GABAergic (GABA) and dopaminergic (DA) [11]. This process, as described by Blum and Kozlowski [10], starts in the hypothalamus with the excitatory activity of serotonin-releasing neurons. This causes the release of the opioid peptide metenkephalin in the VTA, which inhibits the activity of neurons that release the inhibitory neurotransmitter GABA. The disinhibition of dopamine-containing neurons in the VTA allows them to release dopamine in the nucleus accumbens and (via amygdala) in certain parts of the hippocampus, permitting the completion of the cascade and the development of the reward sensation. Usually, if the cascade is working properly, the reward or feeling of "well-being" is obtained provided certain basic conditions are fulfilled. DA is critical to maintain normalization of natural rewards, such as pleasure, peace, stress reduction and wanting [12].

If there is a deficiency or imbalance, the system works abnormally, causing the sense of well-being to be displaced by feelings of 
anxiety, anger, low self-esteem and/or other "bad feelings". Without the normal functionality of DA, an individual will be lacking hedonic response and an inability to cope with stress. The synthesis, vesicle storage, metabolism, release and function of the neurotransmitters are regulated by genes and the expression thereof in terms of messenger RNA directed proteins. So, it is genetically predeterminated whether one individual would have hypodopaminergic activity in central nervous system. These individuals possess a paucity of serotonergic and/or dopaminergic receptors, and an increased rate of synaptic DA catabolism due to high catabolic genotype of the COMT gene. These individuals with hypodopaminergic activity of the brain are predisposed to seek substances and/ or behaviors that will overcome this anhedonic state by activating mesolimbic dopaminergic system. These substances and behaviors include: alcohol, opiates, psychostimulants, nicotine, carbohydrates, cannabinoids, gambling, sex and any excessive pleasure in thrill seeking behavior like video gaming, etc. Acute indulgence in these behaviors leads to release of DA, which overcomes the hypodopaminergic state for temporary relief of discomfort and a "pseudo feeling" of well-being. Unfortunately, chronic abuse of these psychoactive substances and excessive indulgence in the aberrant behaviors leads to inactivation of the brain reward system (neurotransmitter synthesis inhibition, neurotransmitter storage depletion, neurotransmitter depletion, toxic formation of pseudo neurotransmitters and receptor dysfunction). That's why these abusive behaviors ad substance seeking, as ways of providing feel good response, will escalate in craving behavior. Individuals possessing certain genetic polymorphisms (variations) are prone to amplified polymorphic expressions with environmental and lifestyle insult, with an increased risk for impulsive, compulsive and addictive behavior (Reward Deficiency Syndrome) [1,8,23,24].

Dopamine dysregulation syndrome in Parkinson's disease Definition, epidemiology, risk factors

The dopamine dysregulation syndrome (DDS) is a neuropsychiatric behavioral syndrome, characterized by the addictive pattern of dopaminergic drugs intake (in the first place levodopa), taking the medications in large doses greater than those required to treat motor symptoms, despite the development of disabling dyskinesias, as well as severe social destructive behaviors.

The prevalence of DDS was reported in the first study by Giovannoni., et al. [5], as 4.1\%. In this study, 15 out of 364 patients with PD diagnosed with DDS, giving a prevalence of $4.1 \%$. But the prevalence in the general population of patients with PD is currently unknown as the available figures all refer to specialist referral centers with their inherent selection biases. Similar results were seen in an Italian movement disorders clinic, by Pezzela., et al. [19], with 7 out of 202 patients $(3,4 \%)$ who fulfilled the criteria for DDS.

There are some predictive clinical "red flags" for developing DDS in patients with PD. It has been shown that some personality traits have impact on developing DDS. In this group, patients characterized with impulsivity and sensation seeking (ISS), novelty seeking, risk-taking activities, responses to novel stimuli predictive of reward, such as positive affect, increased energy and enhanced psychomotor activity, which are mediated through mesolimbic dopaminergic pathways. (score higher on ISS rating). Further, higher alcohol intake, illicit drug abuse, etc. ISS traits decline with aging, so earlier age of exposure to potentially addictive drugs is known to influence on development of DDS. Patients with PD and DDS are more likely to have a history of depressive symptoms than those who did not develop DDS. These risk factors are clearly presented in the table below (Table 1) [7].

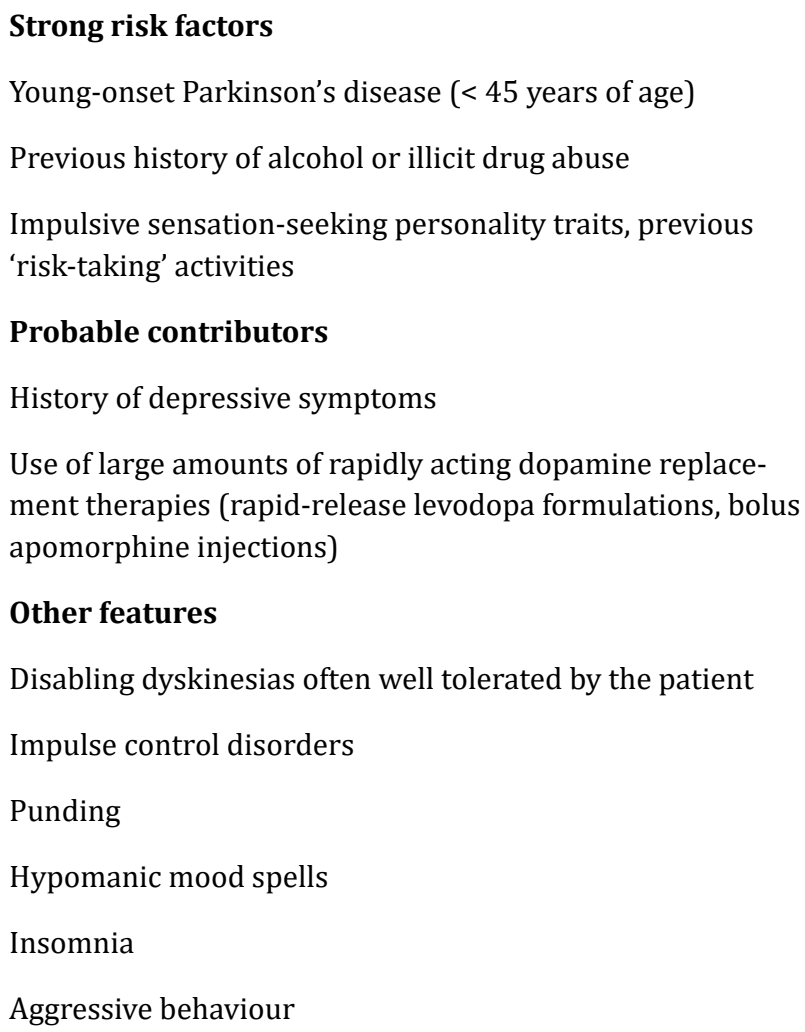

\section{Other features}

Disabling dyskinesias often well tolerated by the patient Impulse control disorders

Punding

Hypomanic mood spells

Insomnia

Aggressive behaviour

Table 1: Clinical 'red flags' for the development of dopamine dysregulation syndrome in patients. 


\section{Pathophysiology in DDS}

The organization of corticostriatal systems has been considered in terms of segregated parallel circuits which connect limbic, prefrontal, oculomotor and motor cortical areas through sub-regions of the basal ganglia and the ventral thalamic nuclei to analogous cortical areas. Functional integration within these pathways is retained by means of neuromodulation occurring through striatal dopaminergic receptors. In PD this modulation is lost due to dorsal striatal dopamine depletion; resultant increased activity in the output nuclei then leads to increased inhibition of the glutamatergic excitation of the motor cortex and a subsequent reduction in voluntary movements. While there is a severe degeneration in the nigral dopaminergic cells, there is a smaller loss of dopamine neurons within the ventral tegmental areas. (Neural activity of ventral tegmental area is associated with the learning of stimuli with behavioral and motivational significance, adaptation of expectations to new contingencies and promotion of attention) [2,16,21,22].

Regarding the pathophysiology of DDS, widely accepted is the incentive sensitization theory of addiction, originally published in 1993. The most important of these psychological changes is a 'sensitization' or hypersensitivity to the incentive motivational effects of drugs. Drug-associated stimuli sensitization produces a bias of attentional processing towards drug-associated stimuli and pathological motivation for drugs (compulsive 'wanting'). When combined with impaired executive control over behavior, incentive sensitization culminates in the core symptoms of addiction $[9,21,22]$.

With repeated exposure to psychostimulants, long-lasting neuroadaptations within the nucleus accumbens [25] and evokes alterations in the reactivity of nerve terminals in the prefrontal cortex [26]. This sensitization is associated with a long-lasting increase in dendritic spine density on medium spiny neurons of both the nucleus accumbens and dorsal striatum [27]. Pathological subversion of normal brain learning and memory processes and enhancement of habit-based learning are proposed to enhance the motivational impact of drug associated stimuli and drive compulsive drug use [28]. Alterations in cortical and limbic inputs to the ventral striatum (arising in the prefrontal, anterior cingulate, hippocampal cortices and amygdala) affect nucleus accumbens function and subsequent outputs to motor relay circuits. In time, drug 'wanting' is thought to become uncoupled from subjective drug 'liking' [9].
Dopamine replacement therapy (DRT) induced neuroplasticity within ventral and dorsal striatal systems and subsequent longterm disruptions of signaling in the basal ganglia, that has been proposed to underlie the behavioral and motor complications of compulsive medication use in Parkinson's disease. Sensitization of ventral striatal networks to DRT and appetitive behaviors may be analogous to the neuroplastic changes in the dorsal striatum thought to contribute to the motor complications of DRT such as dyskinesias and repetitive motor acts [29]. Furthermore, striatal dopamine denervation in Parkinson's disease and cortically-mediated impairments in goal directed function [30,31] may synergistically enhance sensitization to DRT [9].

\section{Clinical phenomenology}

Typical presentation of a patient with DDS in PD is age of onset of parkinsonismus at about 40 - 50 years, usually male gender, and at the middle or advanced stage of the disease. These patients tend to self-medicate and increase their dopamine replacement therapy (DRT), very rapidly, in large steps, and in excess of what would normally be required to control motor symptoms. Individual doses become larger and more frequent, leading to massive total daily doses of levodopa. Attempts to reduce the dose of levodopa are met with strong resistance and are usually unsuccessful (suggesting psychological dependence). By the time DRT misuse (using somatic cues unrelated to Parkinsonian symptoms as excuses) and behavioral disorders become clinically apparent, these patients have usually developed violent and disabling, but well tolerated, drug induced dyskinesias. An interesting feature is the long latency between the onset of L-dopa treatment and apparent addiction, as both patients were maintained on this medication for years before misuse was noted $[5,10,13-15,17]$.

Phenomenology in DDS varies from hypodopaminergic state and syndrome, to the hyperdopaminergic state, on the opposite side of the spectrum. The common disabling clinical symptoms are craving (for additional medication, often in the absence of withdrawal or off states), euphoria/hypomania (feelings of euphoria, omnipotence, invulnerability and inappropriate joy, ricing thoughts and grandiose ideation, increasing inability to distinguish reality from fantasy, insomnia, psychomotor agitation, can occur during the peak of medication effects), dysphoria (feeling of sadness, exaggerated psychomotor slowing, dysphoria, fatigue, anchedonia and apathy, somatic complaints as a result of withdrawal of DRT), appetitive behaviors (hypersexuality, pathological gambling, 
compulsive shopping, eating disorders, as a feature of hippomanic phases), aggression (including irritability, low tolerance of frustration, angry outbursts, use of insulting language and gestures, jealousy, occasional violence), psychosis (delirium, paranoid ideation, fears of persecution, panic attacks and hallucinations, may all be present in patients with DRT intoxication), punding (motor stereotypes which are meaningless, compulsive behavior, with repetitive tasks such as collecting, arranging objects that develop from proponent habits, which are idiosyncratic and depend on an individual occupation, interests and pastimes).

There is dissociation between knowledge and behavior as individuals become unable to control automatic stimulus-response selection mechanisms: patients know that they are disruptive and unproductive, but continue to perform them $[4,5,9]$.

\section{Diagnostic criteria}

Table 2 summarizes the criteria necessary for a diagnosis of DDS, reproduced by Giovannoni., et al [5].

1. Parkinson's disease with documented levodopa responsiveness

2. Need for increasing doses of DRT in excess of those normally required to relieve Parkinsonian symptoms and signs

3. Pattern of pathological use: expressed need for increased DRT in the presence of excessive and significant dyskinesias despite being on, drug hoarding or drug-seeking behavior, unwillingness to reduce DRT, absence of painful dystonias

4. Impairment in social or occupational functioning: fights, violent behavior, loss of friends, absence from work, loss of job, legal difficulties, arguments or difficulties with family

5. Development of hippomanic, manic or cyclothymiacs affective syndrome in relation to DRT

6. Development of a withdrawal state characterized by dysphoria, depression, irritability and anxiety on reducing the level of DRT

7. Duration of disturbance of at least 6 months

Table 2: Diagnostic criteria used to diagnose dopamine dysregulation syndrome.

\section{Management}

The first step is gradually reducing dopaminergic dosages. 'Booster' medications, such as subcutaneous bolus doses of apomorphine and rapid-acting levodopa formulations, should be stopped completely. The patients need to be kept $\mathrm{ON}$ all day long.
So, long acting Levodopa is probably the best way to help these patients.

Hippomanic and psychotic episodes associated with DDS are best managed in a hospital setting and if dose reduction does not improve the situation, antipsychotic medications such as quetiapine, clozapine, should be considered. There are currently no data available regarding the preferred antidepressant types in treating DDS. Rarely, electroconvulsive therapy may be required $[7,9]$.

There are studies which demonstrate the effective use of valproic acid (VA) for the treatment of DDS occurring in PD, without side effects. Valproic acid as an anticonvulsant has multimodal mechanisms of action. Along with lithium and carbamazepine, as mood stabilizers have been claimed to be useful in treatment of these patients. But there is little evidence and experience of these agents in treating patients with DDS in PD [7,20].

In refractory cases, DBS (deep brain stimulation) of subthalamic nucleus is taking in consideration, especially cases with severe motor dyskinesias, but further research is required to identify factors that can predict those patients with DDS, who will derive benefit from deep brain stimulation [7].

If the patients insight into their problem, and agree, enrolment into a drug addiction rehabilitation program is appropriate. The prognosis is generally poor with patients often finding other sources for their medication, with relapse being the inevitable consequence [5].

The opinion of Roberto Cilia., et al. in their study [18] regarding the management of DDS, as a conclusion is: Clozapine and STN-DBS are often mandatory to compensate severe On-Off fluctuations and craving symptoms; antidepressants and/or mood stabilizers may be helpful but are unlikely to lead to remission of addictive behaviors. Effective supervision in every-day life seems to be the most important factor associated with long-lasting DDS resolution.

\section{Conclusion}

Dopamine Dysregulation syndrome (DDS) is a very often unrecognized disorder in patients with Parkinson's disease. It has a very complex pathophysiological mechanism that needed further investigations. Patients with PD that belong to "risk population", should underwent detail interview and psychological testing, on a regular basis, due to revealing DDS on time. Hence we can expect more benefit and more efficiency in applying the recommended measures. 


\section{Bibliography}

1. Kenneth Blum., et al. "Activation instead of blocking mesolimbic dopaminergic reward circuitry is a preferred modality in the long term treatment of reward deficiency syndrome (RDS): a commentary". Theoretical Biology and Medical Modeling 5 (2008): 24.

2. Warren N., et al. "Dopamine dysregulation syndrome in Parkinson's disease: a systematic review of published cases". Journal of Neurology, Neurosurgery, and Psychiatry 88 (2017): 1060-1064.

3. Eric Ch Wolters., et al. "Parkinson's disease-related disorders in the impulsive-compulsive spectrum". Journal of Neurology 255.5 (2008): 48-56.

4. Roberto Ceravolo., et al. "Spectrum of addictions in Parkinson's disease: from dopamine dysregulation syndrome to impulse control disorders". Journal of Neurology 257.2 (2010): S276-S283.

5. G Giovannoni., et al. "Hedonistic homeostatic dysregulation in patients with Parkinson's disease on dopamine replacement therapies". Journal of Neurology, Neurosurgery, and Psychiatry 68 (2000): 423-428.

6. K Blum., et al. "Neurogenetic Impairments of Brain Reward Circuitry Links to Reward Deficiency Syndrome (RDS): Potential Nutrigenomic Induced Dopaminergic Activation". Journal of Genetics and Gene Therapy 3.4 (2012): 1000e115.

7. Sean S O'Sullivan., et al. "Dopamine Dysregulation Syndrome. An Overview of its Epidemiology, Mechanisms and Menagement". CNS Drugs 23 (2009): 157-170.

8. Mahlon R DeLong and Thomas Wichmann. "Circuits and Circuit Disorders of the Basal Ganglia". Archives of Neurology 64 (2007): 20-24.

9. Andrew H Evans and Andrew J Lee. "Dopamine dysregulation syndrome in Parkinson's disease: Editorial Review”. Current Opinion in Neurology 17 (2004): 393-398.

10. Blum K and Kozlowski GP. "Ethanol and neuromodulator interactions: a cascade model of reward. Alcohol and Behavior". Edited by: Ollat H, Parvez S, Parvez H. 1990, Utrecht, Netherlands: VSP Press (1990): 131-149.
11. Malhotra AK., et al. "Genomics and the future of pharmacotherapy in psychiatry". International Review of Psychiatry 19 (2007): 523-530.

12. Carelli RM. "The nucleus accumbens and reward: neurophysiological investigations in behaving animals". Behavioral and Cognitive Neuroscience Reviews 1 (2002): 281-296.

13. Kurlan R. "Disabling repetitive behaviors in Parkinson's disease". Movement Disorders 19 (2004): 433-437.

14. Voon V. "Repetition, repetition and repetition: Compulsive and punding behaviors in parkinson's disease”. Movement Disorders 19 (2004): 367-370.

15. Mia Zaharna MPH and Mayur Pandya. "Levodopa Addiction and Factitious Disorder in a Patient With Idiopathic Parkinson's Disease". The Journal of Neuropsychiatry and Clinical Neurosciences 22 (2010): 3 .

16. Anthony A Grace. "Dysregulation of the dopamine system in the pathophysiology of schizophrenia and depression". Nature Reviews Neuroscience 17.8 (2016): 524-532.

17. Leora L Borek and Joseph H Friedman. "Levodopa addiction in idiopathic Parkinson disease”. Neurology 65 (2005).

18. Cilia R., et al. "Dopamine dysregulation syndrome in Parkinson's disease: from clinical and neuropsychological characterization to management and long-term outcome". Journal of Neurology, Neurosurgery, and Psychiatry 8 (2014): 311-318.

19. Pezzella FR., et al. "Prevalence and clinical features of hedonistic homeostatic dysregulation in Parkinson's disease". Movement Disorder 20.1 (2005): 77-81.

20. Ashok Sriram., et al. "Valproate as a treatment for dopamine dysregulation syndrome (DDS) in Parkinson's disease". Journal of Neurology 260.2 (2013): 521-527.

21. Robinson TE and Berridge KC. "Addiction". The Annual Review of Psychology 54 (2003): 25-53.

22. Robinson TE and Berridge KC. "The psychology and neurobiology of addiction: an incentive-sensitization view". Addiction 95.2 (2000): S91-S117.

23. Xiao C., et al. "Effects of ethanol on midbrain neurons: role of opioid receptors". Alcoholism: Clinical and Experimental Research 31 (2007): 1106-1113. 
24. Blum K., et al. "Reward deficiency syndrome: a biogenetic model for the diagnosis and treatment of impulsive, addictive, and compulsive behaviors". Journal of Psychoactive Drugs 32 (2000): i-iv.

25. Li Y and Kauer JA. "Repeated exposure to amphetamine disrupts dopaminergic modulation of excitatory synaptic plasticity and neurotransmission in nucleus accumbens". Synapse 51 (2004): 1-10.

26. Kalivas PW and McFarland K. "Brain circuitry and the reinstatement of cocaineseeking behavior". Psychopharmacology (Berl) 168 (2003): 44-56.

27. Li Y., et al. "The location of persistent amphetamine-induced changes in the density of dendritic spines on medium spiny neurons in the nucleus accumbens and caudate-putamen". Neuropsychopharmacology 28 (2003): 1082-1085.

28. Robbins TW and Everitt BJ. "Limbic-striatal memory systems and drug addiction". Neurobiology of Learning and Memory 78 (2002): 625-636.

29. Evans AH., et al. "Punding in Parkinson's disease: its relation to the dopamine dysregulation syndrome". Journal of Movement Disorders 19 (2004): 397-405.

30. Cools R., et al. "L-Dopa medication remediates cognitive inflexibility, but increases impulsivity in patients with Parkinson's disease". Neuropsychologia 41 (2003): 1431-1441.

31. Emre M. "What causes mental dysfunction in Parkinson's disease?" Journal of Movement Disorders 18.6 (2003): S63-S71.

\section{Assets from publication with us}

- Prompt Acknowledgement after receiving the article

- Thorough Double blinded peer review

- Rapid Publication

- Issue of Publication Certificate

- High visibility of your Published work

Website: www.actascientific.com/

Submit Article: www.actascientific.com/submission.php

Email us: editor@actascientific.com

Contact us: +919182824667 